\title{
Null Tests of Breakthrough Energy Claims
}

\author{
Scott R. Little* \\ EarthTech International, Austin, Texas, 78759
}

\begin{abstract}
Mankind desperately needs a better source of energy both for space travel and for terrestrial uses. This need spawns invention and claims of new energy devices abound. A number of such devices have been evaluated, with a singular lack of success. Interesting case histories are presented with the goal of promoting a better understanding of the problems encountered in the evaluation of energy devices.
\end{abstract}

\section{Introduction}

$\mathrm{T}$

HERE is no question that our hydrocarbon-based energy system is slowly breaking down. The supply of fossil fuel is finite and combustion products are steadily polluting our environment. Conventional nuclear energy is also problematic: the fuel is still in limited supply, undesirable waste products are produced, and there is significant public concern about the safety of nuclear power plants. But these terrestrial problems pale in comparison to the likely energy requirements for interstellar travel. For example, consider a hypothetical mission to Alpha Centauri in a ship the size of a Boeing 757 (i.e. $10^{5} \mathrm{~kg}$ ). You accelerate at $1 \mathrm{~g}$ to the midpoint and then decelerate at $1 \mathrm{~g}$ until you arrive at your destination 4.3 light-years from Earth. At the midpoint, the ship is moving at $0.95 \mathrm{c}$. For the passengers the trip takes only 3.6 years whereas 5.9 years go by for those who stay on Earth. A yet-to-be-developed engine that converts its fuel entirely to energy and beams it out the tailpipe drives your ship with the maximum possible fuel efficiency. Despite this efficiency, the trip requires a staggering $3.8 \times 10^{6} \mathrm{~kg}$ of fuel: 38 times the weight of the ship. The energy required for this ideal one-way trip to Alpha Centauri is about 800 times the present annual energy consumption of the world.

Faced with such monumental problems humans naturally devise schemes to solve them. Often, the scheme involves a device that is purported to produce more energy than it takes to run it. Sometimes the inventor naively thinks his device is simply creating the extra energy. More often the inventor believes that his device is tapping a new source of energy.

Breakthrough energy claims are nothing new. Recorded history of perpetual motion claims begins in the $13^{\text {th }}$ century with a simple mechanical device attributed to Wilars de Honecort. ${ }^{1}$ Leonardo da Vinci toyed with the idea of a hydraulic perpetual motion machine in his youth. ${ }^{2}$ In the 1700's Johann Bessler, a.k.a. Orffyreus, developed a mechanical perpetual motion machine that was widely witnessed yet remains shrouded in mystery to this day. There are numerous other devices, primarily invented by charismatic individuals with no particular scientific training. As science progressed toward formal recognition of the laws of thermodynamics in the mid 1800's, claims to perpetual motion became considerably less acceptable but hardly less frequent. In 1870, Henry Dircks eloquently described claimants to perpetual motion as follows, "A more self-willed, self-satisfied, or self-deluded class of the community, making at the same time pretension to superior knowledge, it would be impossible to imagine. They hope against hope, scorning all opposition with ridiculous vehemence, although centuries have not advanced them one step in the way of progress." 3

Fortunately, things have changed. In our present age of science and technology almost everybody accepts the laws of thermodynamics. The majority of new energy claims are therefore based upon the idea of tapping a new source of energy. It is these claims that deserve our attention and are the primary focus of this report.

\section{Testing of Energy Claims}

Testing of energy devices is conceptually straightforward. The output energy of the device is measured, the apparent input energy is measured, and the two quantities are compared. If the device is working as claimed, i.e. extracting energy from some unusual source and delivering it to the output, the measured output energy will exceed

*Experimentalist, little@earthtech.org, AIAA member 
the measured input energy. If the device is not working as claimed, the $1^{\text {st }}$ Law of Thermodynamics requires that the output energy, including any losses such as heat, must be precisely equal to the input energy.

In the case of an electrical input, measurement of the input energy is relatively straightforward, particularly if the input is steady DC. If the input has temporal variations, a sophisticated power analyzer ${ }^{4}$ may be required to achieve satisfactory measurement accuracy. In the case of mechanical input like a rotating shaft, a dynamometer, which measures torque and angular velocity, is required to directly measure the mechanical input energy.

Measurement of the output energy is often more challenging. Devices that produce direct electrical output can be handled by the methods outlined above for input energy measurement. Devices that produce heat energy as their output require some form of calorimetry. Calorimetry is conceptually simple but, in practice, a great effort is required to reduce errors, primarily systematic, to acceptable levels. It is especially difficult to achieve accuracy levels better than $1 \%$ relative. Compared to a calorimeter with $1 \%$ relative accuracy, at least an order of magnitude more effort is required to achieve $0.1 \%$ accuracy. ${ }^{5}$

A frustrating situation arises when investigating energy claims. A single null test of the claim often proves almost nothing. Regardless of the circumstances one can always say that the test was not conducted properly, e.g. that the right materials were not used, the apparatus was not assembled correctly, or the planets were not properly aligned. Strictly speaking, an infinite number of null tests are required to disprove a claim whereas a single robust positive test suffices to prove a claim.

With this in mind, the ideal condition under which to test an energy claim is with the full cooperation of the claimant using his original apparatus. The testing proceeds rapidly and, in the event of null results, the claimant can ensure as far as possible that the tests are conducted properly. If the original apparatus is not available, a new apparatus must be constructed. In this case, even with the full cooperation of the claimant, there is more room for excuses should the tests yield null results. If the claimant is non-cooperative and the apparatus must be constructed from various documents and records, very little can be proven if the tests are null.

\section{Some Tests of Breakthrough Energy Claims}

This section contains accounts of some of our tests of breakthrough energy claims. As noted above, the fact that our test results were null does not disprove these claims. We present these cases primarily to promote better understanding of the problems involved in such testing.

\section{A. Zero-Point Energy Devices}

Quantum physics predicts the existence of an electromagnetic zero-point field whose energy density is so large that many physicists, unable to accept it as physically real, designate it as virtual. Others accept the zero-point field as real energy that surrounds and pervades everything leaving ordinary matter as nearly negligible foam riding on this vast sea of energy. According to John A. Wheeler, “...elementary particles represent a percentage-wise almost completely negligible change in the locally violent conditions that characterize the vacuum.” ${ }^{\text {This viewpoint has }}$ led to intense speculation about the possibility of utilizing zero-point energy.

There is a real force that can be attributed to the zero-point field: the Casimir force. ${ }^{7}$ Experimentally confirmed, ${ }^{8}$ this force arises when conductive surfaces are placed in very close proximity, thus creating a cavity that eliminates certain electromagnetic modes between the plates. The result is an imbalance in the radiation pressure on the two sides of each plate, ${ }^{9}$ which produces a net force that pushes the plates together. Some physicists, including Robert Forward, ${ }^{10}$ have proposed that the Casimir force provides a means of extracting energy from the zero-point field. Julian Schwinger ${ }^{11}$ provided further stimulus by suggesting that the energy released in sonoluminescence was due to Casimir forces acting on the collapsing bubbles.

Our first and most extensive campaign to extract energy from the zero-point field was an effort to replicate the energy claims associated with Ken Shoulder's charge clusters, or EV's as they are popularly known. Shoulders believes that at least one EV is formed in every spark discharge. EV's are supposed to contain about $10^{9}$ electrons and exist only during the transit from cathode to anode. Sharply pointed cathodes and a fast rise time for the applied high voltage pulse promote EV formation. The connection with zero-point energy comes from the hypothesis that the compression of electrons into a charge cluster is due to attractive Casimir forces overpowering repulsive Coulomb forces at very short range. Shoulders did a great deal of experimentation with EV's and claimed in US 
Patent 5,018,180 (May 21, 1991) to have observed 96 times more energy released by an EV than required to produce the EV. We pursued this claim for years and were never able to reproduce Shoulder's results. We experimented with numerous setups in an effort to observe the direct electrical energy output that Shoulders had claimed in his patent. Failing that, we attempted calorimetric measurements that were of compounded difficulty because of the low energy levels involved, the difficulty of accurately measuring the input energy delivered to the spark discharge, and the overall difficulty of making sensitive measurements in such an electrically noisy environment. Despite these problems we eventually managed to obtain reasonable accuracy and reliability in our calorimetric measurements of EV's and the results were disappointingly negative.

Schwinger's hypothesis led us to give serious consideration to several cavitation-based energy claims. The Potapov device, invented by Yuri Potapov in Moldavia is an example. The Potapov device consisted simply of a swirl chamber through which water was pumped vigorously to create a vortex and cavitation. Potapov claimed that his device imparted to the water up to 3 times more heat energy than the mechanical energy required to pump the water through it. In this case we were able to obtain a genuine Potapov device for testing and we had limited cooperation from Potapov himself. We constructed a batch calorimeter system in which the device would be operated for a certain period of time to heat up the water contained in a large insulated reservoir. Water was pumped from the reservoir, through the device, and returned to the reservoir. The pump was driven by an electric motor. For the input energy we simply measured the electrical energy required to drive the motor using a 3-phase watthour meter. For the output energy we measured the increase in water temperature and used the total weight of water in the tank to compute the heat energy delivered to the water. Instead of the $300 \%$ efficiency claimed for the Potapov device we observed only $80 \%$ at best. As a control, we also measured the heating efficiency of a simple gate valve inserted in the flow path in place of the Potapov device and adjusted to provide about the same flow restriction. The valve heated the water just as efficiently as the Potapov device. The testing went on for months as we struggled to communicate with Potapov. He did not think we were operating his device properly and we made numerous modifications at his request. The test results remained uniformly negative.

Another device (which must remain nameless due to a non-disclosure agreement with the developer) involved a motor-driven rotor in a close-fitting housing. Water was forced through the gaps around the rotor where intense cavitation was supposed to occur. This device was claimed to impart up to $50 \%$ more heat energy to the water flowing through it than the mechanical energy required to drive it. We tested this device using a larger version of the same batch calorimeter described above. In this case we constructed a cradle dynamometer to directly measure the mechanical input energy. With a $30 \mathrm{hp}$ electric motor and copious generation of steam by the device, this was a very exciting and sometimes dangerous experiment. However, our measurements never showed any sign of excess energy. Furthermore, by comparing mechanical input energy to heat output energy we were able to obtain a nearperfect energy balance in our measurements, typically $99 \%+/-1 \%$. We were fortunate to have significant cooperation from the developer of this device and, during a visit to our facility, we accidentally discovered the source of most of his anomalous readings: improper usage of his electrical power analyzer.

A related claim is that of sonofusion made primarily by Roger Stringham. ${ }^{12}$ We first investigated this claim by constructing our own apparatus without any cooperation from Stringham. The apparatus consists of an ultrasonic transducer immersed in heavy water with a Pd target in close proximity. According to Stringham, more heat energy is produced in the apparatus than the acoustic energy delivered by the transducer. We employed a water-flow calorimeter to measure the heat output energy and we spent a great deal of effort to learn how to accurately measure the high-voltage, $20 \mathrm{kHz}$, low power-factor electrical input energy being delivered to the piezoelectric transducer. To our surprise, a digital oscilloscope that could multiply voltage and current together in real time and integrate the resulting power trace was usually in error by at least $10 \%$ relative. A medium-quality power analyzer, whose specifications indicated that it should achieve $2 \%$ accuracy, was found to be in error by about $20 \%$ relative. Finally we discovered the Clarke-Hess 2330 power analyzer, which appears to actually achieve its accuracy specification of $+/-0.2 \%$. We conducted a total of 48 runs with our apparatus, 12 of which used Pd targets. We never saw any sign of excess heat. After communicating our results to Stringham we arranged to visit his laboratory with a portable version of our calorimeter and our Clarke Hess 2330. In other words, we were given a chance to test his claim using his apparatus with his full cooperation. We succeeded only in demonstrating that his input power measurements were erroneous and understating the actual input power. That was the cause of his apparent excess heat on that day in 1999. Today, Stringham is exploring this phenomenon with considerably reworked apparatus and is claiming even higher excess energy production. We look forward to examining his claims again. 
EarthTech colleague Hal Puthoff ${ }^{13}$ has shown that the ground state of the hydrogen atom can be explained as a dynamic balance between energy lost by the electron due to acceleration radiation and energy absorbed from the zero-point field. The fact that the space between Casimir plates is a region where the zero-point field is reduced in energy density led to speculation that hydrogen might lose some of its ground state energy when placed in such a cavity. If that were the case, then that energy release would constitute zero-point energy conversion and a circulation of hydrogen into and out of a Casimir cavity might produce a continuous extraction of energy from the zero-point field. We have designed and constructed several experiments to explore this hypothesis but without success so far. Most of them were attempts to detect heat energy being released by hydrogen flowing through some form of Casimir cavity. We first tried constructing cavities out of precision optical flats. For the ground state of molecular hydrogen, the optimum cavity spacing is about 1 micrometer. We carefully monitored the gas temperature at the entrance and exit of the cavity looking for signs of heating due to the release of ground state energy. We actually did observe a small temperature increase in this experiment but it turned out to be due only to the Joule-Thompson effect which, for hydrogen, results in a warming of the gas as it flows through a restriction. We also tried using finely powdered metals such as Pt and Pd to create a dense labyrinth of Casimir scale passages. These experiments tended to produce an exciting initial burst of heat when the flow of $\mathrm{H}_{2}$ was started. But the burst always faded away after a minute or two and it could not be readily repeated. We finally tracked this down to $\mathrm{H}_{2}+\mathrm{O}_{2}$ combustion catalyzed by the finely powdered metal. It was not readily repeatable because the apparatus was nearly sealed. Only after the apparatus had been sitting overnight or had been disassembled was there sufficient $\mathrm{O}_{2}$ present for another heat burst.

In a different approach to testing this hypothesis we put hydrogen molecules in a Casimir cavity and used absorption spectroscopy to look for a shift in their ground state energy. We performed this experiment at the Synchrotron Radiation Center of the University of Wisconsin-Madison. Following in the footsteps of molecular spectroscopy pioneer Gerhard Herzberg, ${ }^{14}$ we used extreme ultra-violet radiation to probe the dissociation energy of $\mathrm{H}_{2}$ molecules in an appropriate Casimir cavity. We assumed that a depression of the ground state energy would produce a corresponding increase in the dissociation energy. A great deal of effort went into the apparatus for this experiment and we encountered severe problems that forced us to rebuild part of the experiment while at the synchrotron facility. Unfortunately we did not find evidence of the ground state shift, a result we presented at the International Conference on Squeezed States and Uncertainty Relations (ICSSUR 2001), Boston University, 2001. As usual, the possibility remains that such ground state shifts do occur but that this particular experiment did not reveal them. Further effort along this line by a consortium of researchers is planned. ${ }^{15}$

\section{B. Electromagnetic Devices}

A number of electromagnetic energy claims have been made over the past 150 years. Some of them are no more than a continuation of the quest for perpetual motion but with magnets and coils replacing the weights and levers of the earlier devices. Others, particularly the more recent claims, are not so easy to dismiss and deserve to be investigated.

A relatively simple device called the Motionless Electromagnetic Generator (MEG) (US Patent \#6,362,718 March 26, 2002) has been widely publicized on the Internet. We constructed our own MEG using detailed construction plans from an independent lab that reportedly had successfully replicated the excess power results. The initial results with our MEG also apparently showed excess power but we soon learned why. The MEG operates at high audio frequencies and delivers several hundred volts and a few milliamperes to a load resistor. This current was being measured using a 10-ohm current-viewing resistor in series with the load resistor. With only a few milliamperes of current through this resistor, the voltage developed is only a few tens of millivolts. At these frequencies it is almost impossible to accurately measure this small voltage while in intimate proximity to the output voltage, which is 4 orders of magnitude higher. Capacitive coupling between the voltmeter or scope leads significantly elevated the observed voltage across the current viewing resistor resulting in overestimation of the output power. Supporting this conclusion was the lack of heating in the load resistor that should have occurred had the apparent output power been correct. When we changed the current viewing resistor to $1000 \mathrm{ohms}$, which did not significantly affect the load impedance, the apparent excess power disappeared.

The essence of another case, again covered by non-disclosure agreement, can be presented as a good example of an axiom that we have come to embrace: the fact that your instruments cost a lot of money does not guarantee that their results will be accurate. A simple device was being driven by $60 \mathrm{~Hz}$ AC power. The output of this device was also $60 \mathrm{~Hz}$ AC but at a different voltage. We employed our Clarke-Hess 2330 power analyzer for the input and 
output power measurements and obtained a mundane efficiency of $\sim 90 \%$ for the device. However, the claimant was using a state-of-the-art high-bandwidth digital oscilloscope with extensive waveform math capabilities (including power calculation) and a sophisticated clamp-on AC/DC current probe. Surprisingly, this $\$ 30,000$ collection of modern equipment, while capable of accurate power analysis on a wide variety of waveforms over an impressive range of frequencies, made significant errors in the measurement of power in these $60 \mathrm{~Hz}$ signals.

\section{Cold Fusion}

In March of 1989 Martin Fleischmann and Stanley Pons of the University of Utah announced that they had succeeded in making D-D fusion occur in an electrochemical cell near room temperature. Compared to the ordinary conditions required for this reaction, this claim was aptly named "cold fusion". The announcement of cold fusion generated intense interest as it promised to solve most if not all of our energy problems here on Earth. The fuel is plentiful and the waste products are relatively benign. However, widespread failure to replicate the experiment soon resulted in rejection of cold fusion by the mainstream scientific community.

Despite this rejection a number of scientists continue to investigate cold fusion. Hundreds of papers reporting positive results have been published and international conferences are held every couple of years. However, to this day, there exists no cold fusion demonstration experiment. That is because cold fusion phenomena are extremely difficult to reproduce. This situation greatly hampers cold fusion research because it makes the usual empirical investigation almost impossible.

The primary signature of cold fusion is excess heat, which means that the electrochemical cell produces more heat power than the electrical power used to stimulate it. Thus calorimetry is often involved in testing cold fusion experiments. In our laboratory we have expended a great deal of effort on the development of calorimeters suitable for cold fusion experiments. Over the years we have had the opportunity to test a relatively small number of cold fusion cells, some that we constructed ourselves and some that were brought to our laboratory by other investigators who had seen positive signs of excess heat in their own labs. None of these cold fusion experiments have shown any convincing evidence of excess heat in our calorimeters. We cannot say that we have never seen any signs of excess heat in our laboratory because all calorimeters drift somewhat and, inevitably, that drift sometimes goes in a positive direction and looks just like a low level of genuine excess heat. When that occurs we strive to check the calorimeter's calibration as quickly and thoroughly as possible. Usually the drift in calibration is evident and its magnitude matches, and thus explains, the apparent excess heat signal. In a few cases the calibration check did not explain the apparent excess heat signal. But when we returned the cell to the calorimeter after the calibration check, the excess heat signal did not reappear. This tantalizing behavior either means that the cell did produce low levels of excess heat for a while or the calorimeter was simply drifting up and down in unfortunate synchrony with our observations.

In our laboratory, we are not novices at making measurements. We have about 70 years combined experience designing, building, and operating various measuring systems, gauges, and analytical instruments. In addition we have constructed at least a dozen calorimeter systems over the past 15 years in our quest to identify new energy sources. From this broad perspective it seems safe to say that calorimetry excels at providing a fertile medium for the spawning and nurturing of subtle systematic errors. Furthermore we have found it nearly impossible to anticipate the causes of these errors. Their elucidation usually occurs only after you have constructed the instrument, tested it extensively, and struggled for days to comprehend its misbehavior.

The culmination of our efforts to build an accurate and reliable calorimeter for cold fusion experimentation is an instrument we call MOAC (Mother Of All Calorimeters). This instrument operates on a simple and fundamental principle. Flowing water is used to extract the heat from the cell. The flow rate is measured and the temperature rise of the water is measured. The product of the temperature rise, the flow rate, and the specific heat of water yields the heat power being extracted from the cell. Despite its simple concept, MOAC is not a simple instrument. Two independent computer-based data acquisition systems monitor a total of 45 parameters, including 22 temperatures. Fourteen analog outputs, driven by proportional-derivative feedback algorithms, control various critical parameters.

The cell and heat exchanger are located in a chamber whose walls are made almost perfectly insulating by a system that heats the outer surface of each of the six wall panels so that its temperature matches that of the corresponding inner surface. This active insulation ensures that virtually all of the heat dissipated by the cell leaves the chamber via the flowing water. A cascade of three independent Peltier regulators controls the temperature of the 
water entering the heat exchanger to $+/-0.0003^{\circ} \mathrm{C}$. A positive-displacement pump driven by a synchronous motor powered by a crystal-based oscillator produces an exceedingly stable flow of about $2.5 \mathrm{gm} / \mathrm{s}$. A flowmeter consisting of an automated batch weighing system measures the flow rate periodically and typically reports a standard deviation of only $+/-0.0002 \mathrm{gm} / \mathrm{s}$ (i.e. $0.01 \%$ relative). A large well-insulated enclosure houses the entire system. Air circulates over the calorimetry apparatus and then is ducted to a Peltier air-conditioner where its temperature is regulated to $+/-0.001^{\circ} \mathrm{C}$ before it re-enters the enclosure.

MOAC was designed to achieve $+/-0.1 \%$ relative accuracy. At the typical input power level of 10 watts, that is equivalent to +/- 0.01 watts. On a good day, when freshly calibrated, this accuracy is actually achieved. A month after calibration, the system typically drifts by up to 0.03 watts. We believe this drift originates primarily in the thermistors used to measure inlet and outlet water temperatures. Despite this small problem, we feel that MOAC is one of the best calorimeters now available for cold fusion research. The space available for the cell is relatively large (about $10 \mathrm{~cm} \times 25 \mathrm{~cm} \times 25 \mathrm{~cm}$ ). The cell sits in a stirred air environment where it is not thermally clamped to a specific temperature. MOAC exhibits excellent specimen versatility by producing precisely the same reading regardless of the size, shape, or location of the heat source. We are committed to maintaining MOAC in top working condition on a continuous basis. In the interest of scientific progress, we have made a standing offer for free testing of promising cold fusion cells in MOAC.

\section{Conclusion}

Testing of breakthrough energy claims is simple in concept but often difficult in practice. It is most effectively done with the full cooperation of the claimant. Systematic errors are common. Considerable diligence is required to ensure that the measurement techniques employed are acceptably free from such problems.

We hope that this brief account of our own experiences will be of some use to other investigators. For additional information, please address correspondence to Scott Little, EarthTech International, Inc., 4030 Braker Lane West, Austin TX 78759, or electronic mail to little@earthtech.org.

\section{References}

\footnotetext{
${ }^{1}$ Dircks, Henry, Perpetuum Mobile, E. \& F.N. Spon, 1870, p.1.

${ }^{2}$ Heaton, Mrs. Charles W., Leonardo Da Vinci and His Works, Kessinger Publishing, 2004, pp. 154-155.

${ }^{3}$ Dircks, Henry, Perpetuum Mobile, E. \& F.N. Spon, 1870, p.354.

${ }^{4}$ A typical electrical power analyzer samples the voltage and current being delivered to a device rapidly and simultaneously. Each pair of samples is multiplied together to obtain a measure of the instantaneous power flowing into the device. These values are then integrated over time to obtain the electrical energy consumed by the device.

${ }^{5}$ McCullough \& Scott, "Experimental Thermodynamics, Calorimetry of non-reacting systems”, p.9, Vol. 1, 1968,

${ }^{6}$ Wheeler, John A., “Geometrodynamics”, Academic Press (1962).

${ }^{7}$ H. B. G. Casimir, “On the attraction between two perfectly conducting plates,” Proc. Kon. Ned. Akad. Wetensch. 51, 793-796 (1948)

${ }^{8}$ Harris, B.W., Chen, F., Mohideen, U., "Precision measurement of the Casimir force using gold surfaces," Physical Review A, vol 62 (2000). P.052109/1-5.

${ }^{9}$ Milonni, P. W., Cook, R. J., and Goggin, M. E., "Radiation pressure from the vacuum: Physical interpretation of the Casimir force," Physical Review A, vol. 38, p. 1621 (1988)

${ }^{10}$ Forward, Robert L., "Extracting electrical energy form the vacuum by cohesion of charged foliated conductors," Physical Review B, vol 30, number 4, 15AUG1984, p.1700.

${ }^{11}$ Schwinger, Julian, “Casimir light: The souce”, Proc. Natl. Acad. Sci. USA, Vol 90, pp.2105-2106, March 1993

${ }^{12}$ R. S. Stringham, D. R. George, F. L. Tanzella, M.Williams, “Cavitation-Induced Heat in Deuterated Metals,” EPRI Report TR108474, March 1998.

${ }^{13}$ Puthoff, H.E., “Ground State of Hydrogen as a Zero-Point-Fluctuation-Determined State,” Physical Review D, vol. 35, p.3266 (1987)

${ }^{14}$ Herzberg, G., “The dissociation energy of the hydrogen molecule”, Journal of Molecular Spectroscopy, vol 33, pp. 147-168 (1970).

${ }^{15}$ E. W. Davis, et al. (2006), "Review of experimental concepts for studying the quantum vacuum field," Proc. of the STAIF2006: 3rd Symposium on New Frontiers and Future Concepts, AIP Conf. Proc., vol. 813, ed. M. S. El-Genk, AIP Press, pp. 1390-1401.
} 